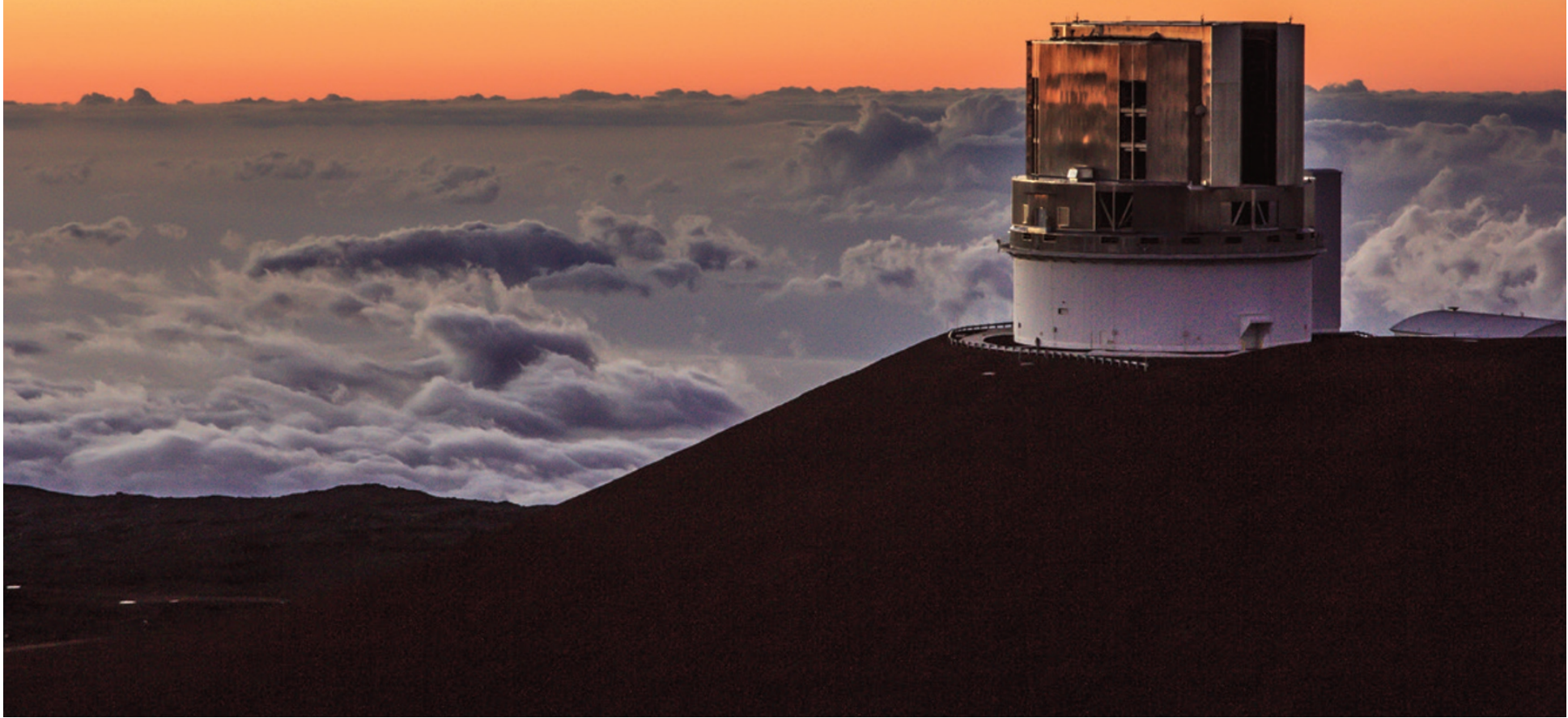

The Subaru telescope in Hawaii, managed by NINS, was integral in the discovery of five new planets and is a fine example of the benefits of international collaboration.

\title{
TOP TEAMS TO BE RECKONED WITH
}

\author{
Japan's institututions are increasingly joining huge collaborative research efforts, \\ realizing that, on the big questions facing science, a problem shared is a problem halved.
}

\section{BY SMRITI MALLAPATY, SIMON PLEASANTS AND NICKY PHILLIPS}

$\mathrm{T}$ hese days, large-scale international collaborations are a key engine of cutting-edge science, and the past 12 months have provided some prime examples. While a single paper in Physical Review Letters credited more than 5,000 researchers, breaking the record for the most authors on a single article, the 2016 Breakthrough Prize in Fundamental Physics was shared between 1,377 scientists investigating neutrino oscillations.

The Nature Index is well poised to evaluate this important phenomenon because it counts the number of authors from different institutions who contribute to every paper included in the index. This is used to assign a collaboration score to institutions and countries. In Japan, the data confirms the link between quality science and collaborations. Four of the top five collaborating institutions in 2015 are also among the most prestigious - the University of Tokyo, RIKEN and the universities of Kyoto and Osaka. Rounding out the top five is the National Institutes of Natural Sciences (NINS), established by the government in 2004 to manage expensive public facilities. In 2014, almost 9,000 researchers from more than 50 countries used the facilities at NINS to conduct research. "This model of designating special institutes to care for large-scale and cutting-edge facilities shared by researchers is unique to Japan," says Amane Koizumi, a professor at NINS. Similar institutes are set up for large information systems and high-energy particle accelerators.

The prominence of NINS reflects how necessity is driving Japanese research institutions to seek collaboration. The 2016 budget for science and engineering is set at 3.45 trillion yen, down $6.4 \%$ from its peak in 2012 . To help institutions cope, the government has introduced policies and programmemes to foster collaboration and resource sharing among research institutes and private companies.

Yasuo Miake, a vice president and executive director for research at the University of Tsukuba, says another factor driving the collaboration effort in Japan institutions is government pressure to contribute to social innovation. There has been a mindset among some Japanese professors that universities should conduct science for science's sake and remain independent of government and industry, says Miake. "That mentality is changing now."

A government strategy released in 2013 identified collaboration between industry, academia and government as one of six essential principles for science policy, setting targets to double, by 2030, the number of joint projects larger than 10 million yen and projects spanning more than three years. Among the institutions whose partnership with industry lead to high-impact research, the National Institute of Advanced Industrial Science and Technology (AIST) received the index's second-highest collaboration score for its partnership with industry in Japan in 2015, following the University of Tokyo. 


\section{COLLABORATION CHAMPIONS \\ Japan's institutions are not just reaching out to domestic partners, but also internationally. The country has a wide collaboration network of academic and corporate connections across the world.}

Top 5 collaborators with companies

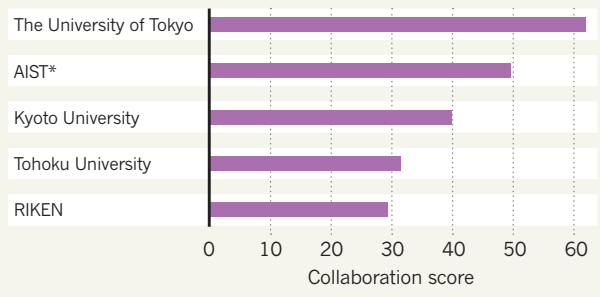

The contribution of the top 10 based on all bilateral partnerships between Japanese institutions and their domestic and international counterparts in 2015

High Energy Accelerator Research Organization University of Tsukuba

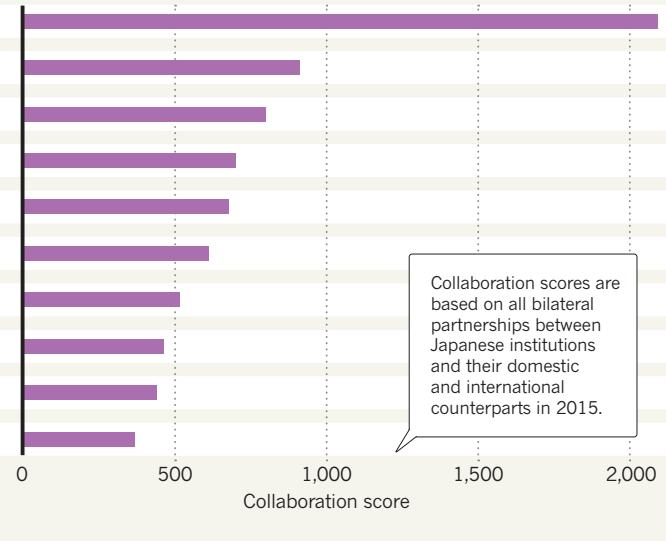
collaborators to Japan's total collaboration score.

*National Institute of Advanced Industrial Science \& Technology (AIST)

\section{RIKEN}

\section{COLLABORATION SCORE: 801}

RIKEN is one of Japan's leading research institutions with roots in basic research, and wants to broaden its connection with industry. It is third on the list of Japanese institutions with the highest collaboration scores in 2015.

"RIKEN is a pure research organization, and one of our strengths is managing large projects and large facilities. These endeavours require collaboration," says a RIKEN executive director, Shigeo Koyasu.

RIKEN's focus on basic science has led to strong partnerships with other domestic and international universities with strengths in this type of research. In the index, RIKEN's top two domestic collaborators are the basic research powerhouses, University of Tokyo and Kyoto

"One of our strengths is managing large projects and large facilities. These endeavours require collaboration." University, with which it works closely on stem-cell science.

RIKEN's top international collaborators in the index are MIT in the United States and the Max Planck Society in Germany, but the institution also has major partnerships with universities in China, Korea, India and Malaysia.

Despite having roots in fundamental research, RIKEN is moving towards solidifying partnerships with industry, says Akihiro Fujita, the head of RIKEN's Cluster for Industry Partnerships. "People in industry have not been sure how to approach RIKEN precisely because our work is in basic research, and this uncertainty may have tended to hinder

\section{RIKEN'S PARTNERSHIPS}

RIKEN's top 5 international collaboration hotspots; and RIKEN's top 5 collaborating institutions.
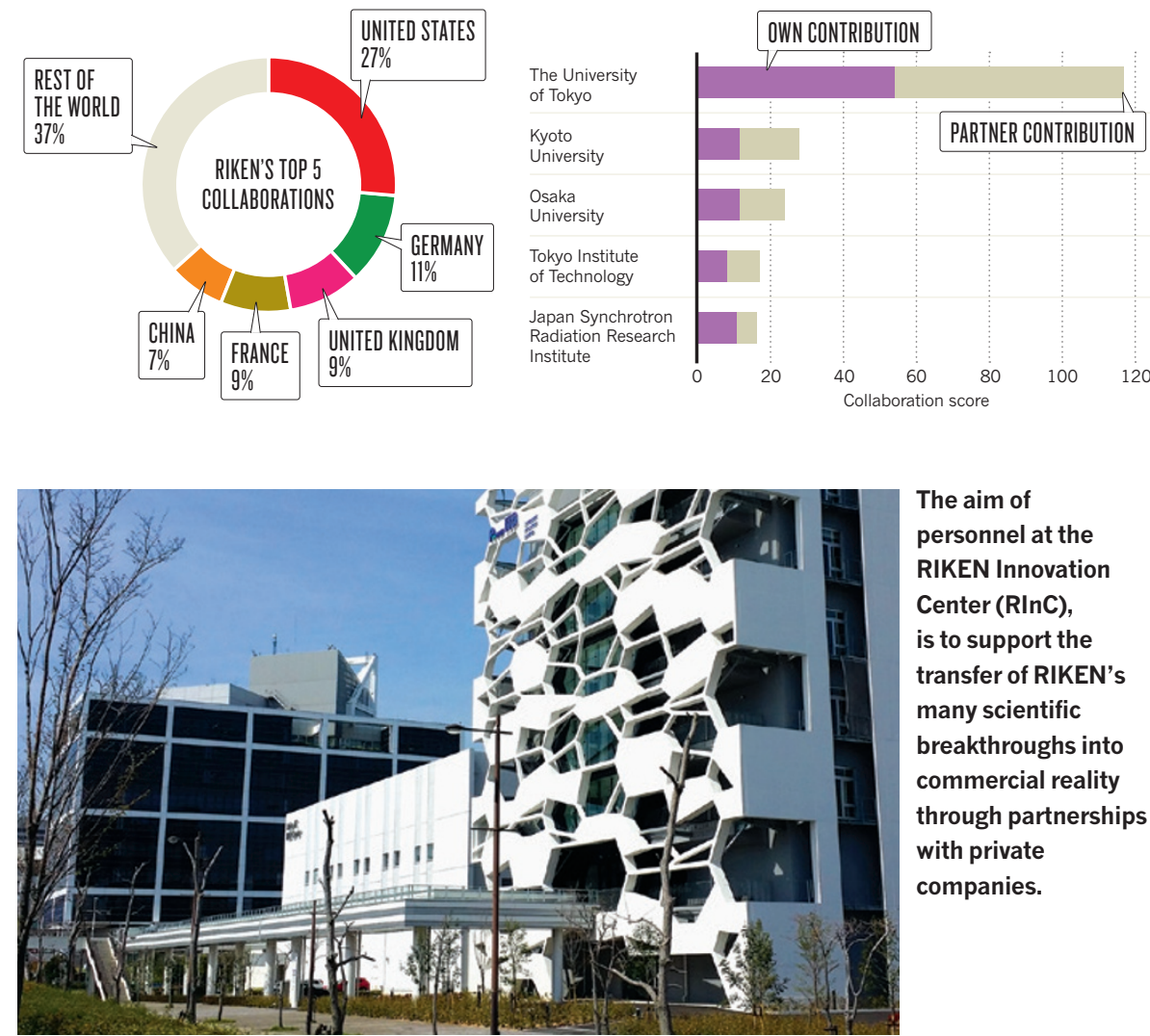

The aim of personnel at the RIKEN Innovation Center (RInC), is to support the transfer of RIKEN's many scientific breakthroughs into commercial reality through partnerships with private companies.

progress in establishing joint research projects with industry." The Cluster for Industry Partnerships aims to bridge that divide by encouraging partnerships with the private sector.

One member of this cluster, the RIKEN Innovation Center, supports researchers to work with private companies to transfer their science into commercial products and services that solve a specific technological challenge. In addition, RIKEN's five collaboration centres with Olympus, Toyota, Rigaku Corporation, Takeda Pharmaceutical and JEOL are expected to lead to new products from basic research findings. 


\section{UNIVERSITY OF TSUKUBA}

\section{COLLABORATION SCORE: 366}

An hour by train north of Tokyo, the University of Tsukuba has taken a structural approach to fostering collaboration. Among the newer national universities, Tsukuba has broken away from some traditional university practices that would have discouraged collaboration. In the past, academic faculties at some universities were organized into large hierarchical research units around a professor. At Tsukuba, groups are much smaller and there are no restrictions on younger assistant or associate professors becoming group leaders, or principal investigators. "Because each research group is small, PIs have to look for outside collaborators to carry

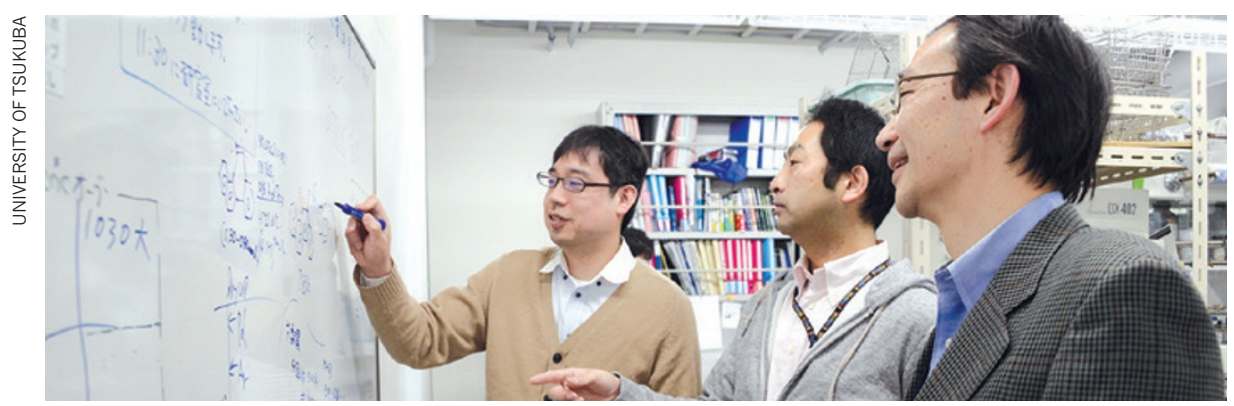

Synthetic organic chemists at Tsukuba University are among the small research groups at the institution. out their research," explains Yasuo Miake, a vice president and executive director for research at the University of Tsukuba. "Big and traditional universities have tremendous barriers between departments. Such barriers are very small at the University of Tsukuba, which allows researchers to talk more easily across departments," he term goal of doubling its income from industry through expanded partnerships with companies such as Toyota, Miake says there is more to achieve. Tsukuba is working to strengthen ties with the almost 100 industrial, academic and governmental organizations clustered at nearby Tsukuba Science City, some of which, like the National Institute of Advanced Industrial Science Technology (AIST), are already among Tsukuba's top domestic collaborators in the index. says. While the university has achieved a short-

\section{NATIONAL INSTITUTES OF NATURAL SCIENCES}

\section{COLLABORATION SCORE: 701}

It took a team of 16 researchers from seven institutions, using three telescopes on two islands to discover five new Jupiter-like planets orbiting three large stars. The findings, published in The Astrophysical Journal, resulted from a 16-year collaboration between the National Astronomical Observatory of Japan, part of the National Institutes of Natural Sciences (NINS), and colleagues in Japan and the United States. Lead

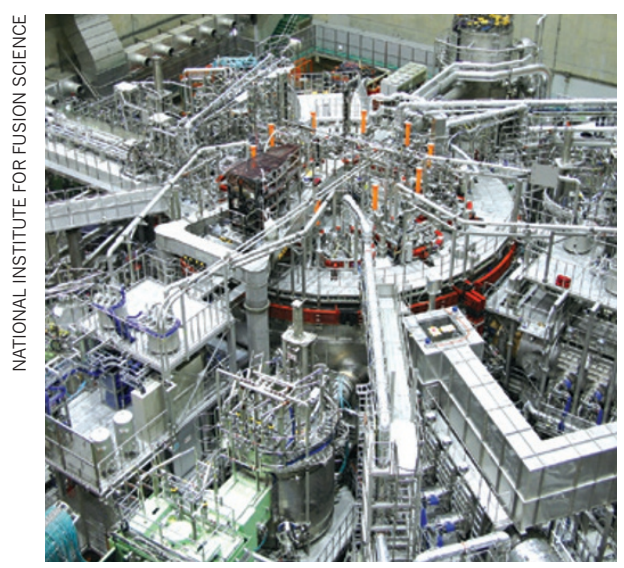

NIFS' Large Helical Device for fusion plasma research author Hiroki Harakawa from NAOJ says in radial-velocity planet searches, collaborating with researchers using other facilities adds to the number of observations required to confirm planetary systems among hundreds of targets. In this case, the observations were made using a telescope in Japan and two telescopes in Hawaii, one which belongs to the United States and the other to Japan, and managed by NINS. Among the many big research facilities it operates, the optical-infrared Subaru telescope on top of a volcano in Hawaii is the jewel in its crown.

NINS's international research alliances may

\section{UNIVERSITY OF TSUKUBA'S PARTNERSHIPS}

University of Tsukuba's top 5 international collaboration hotspots; and top 5 collaborating institutions.
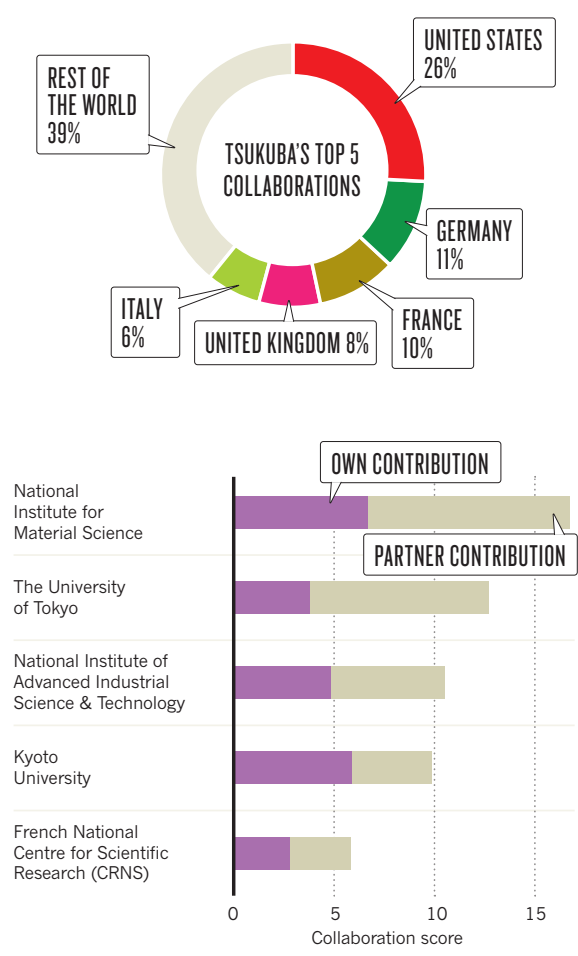

continue to strengthen in the coming decade if the proposed construction of a new 30-metre telescope on the same peak in Hawaii goes ahead. A joint project by institutes in India, the United States, China, Canada and Japan, it would be the world's largest optical-infrared telescope .

In 2015, NINS researchers teamed up with scientists at 490 international and 105 domestic institutions to publish papers featured in the Nature Index, a decline from 2014. Their main domestic collaborator was the University of Tokyo, while the Max Planck Society was their most successful international partnership.

\section{NINS'S PARTNERSHIPS}

NINS's top 5 international collaboration hotspots; and top 5 collaborating institutions.
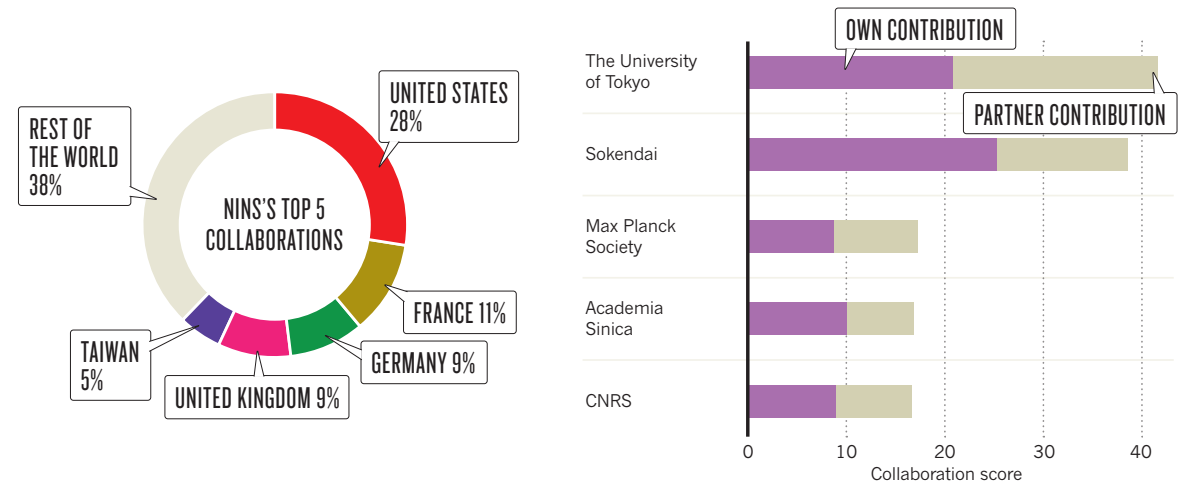


\section{HIGH ENERGY ACCELERATOR RESEARCH ORGANIZATION (KEK)}

\section{COLLABORATION SCORE: 439}

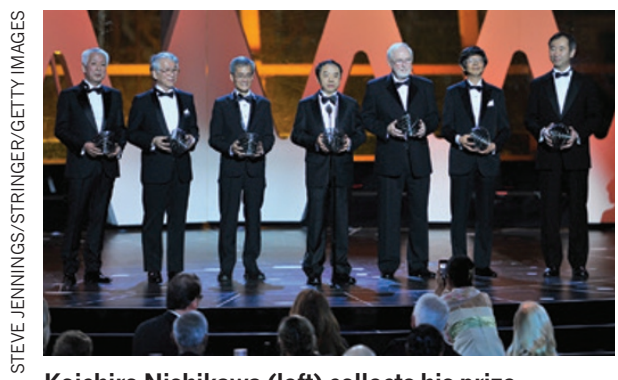

Koichiro Nishikawa (left) collects his prize.

When KEK professor, Koichiro Nishikawa, accepted this year's Breakthrough Prize in Fundamental Physics, he was doing so on behalf of one of five teams awarded for their neutrino experiments that spanned several decades.

Nishikawa is a member of the T2K particle physics experiment, a successor to the K2K programme, a long-baseline neutrino oscillation experiment involving collaborations between several countries including Japan, Germany, Italy, South Korea, Spain, the United States and the United Kingdom. These countries represent seven of the top ten nations with whom Japan

\section{KEK'S PARTNERSHIPS}

KEK'S top 5 international collaboration hotspots; and top 5 collaborating institutions.
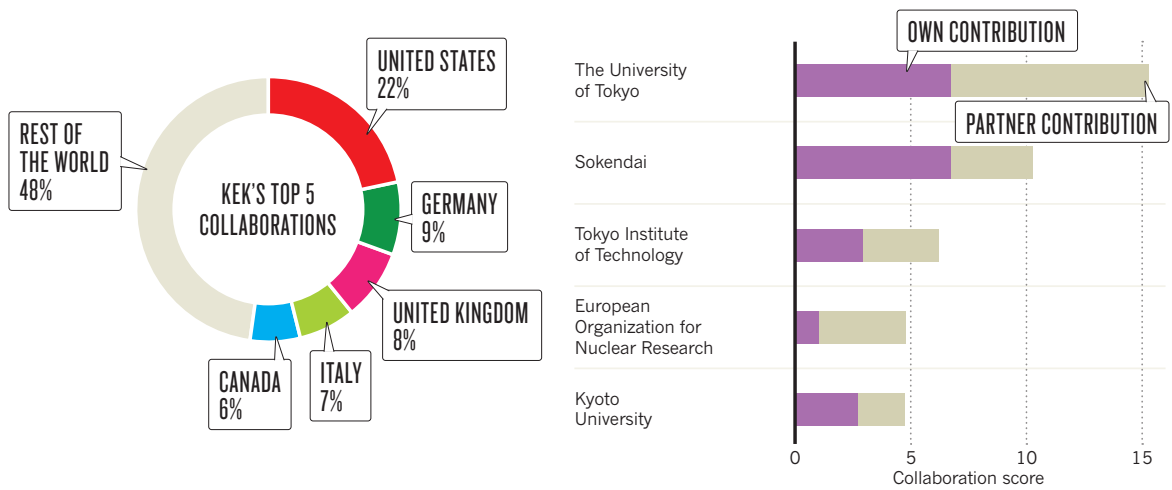

partnered to produce high-quality science included in the Nature Index in 2015.

Another huge and successful collaboration involving KEK scientists is the ATLAS experiment, which observed a particle that behaved exactly like the illusive Higgs boson in 2012.

About 200 Japanese scientists, including 14 from $\mathrm{KEK}$, were involved in major elements of the construction of the ALTAS detector, including the silicon strip detector to detect charged particles, the superconducting solenoid magnet and the electronics for the muon trigger chamber. "To build the large accelerator and the large detector, international collaboration is essential in terms of both budget and human power," says Kazunori Hanagaki, professor of the Institute of Particle and Nuclear Studies (IPNS) at KEK.

In the Nature Index, KEK's focus on highenergy physics reflects the main institutions with which its researchers collaborate. KEK scientists co-authored the most high-impact articles with counterparts at the INFN National Laboratory in Italy, publishing 141 papers in the 68 highimpact journals featured in the Nature Index.

\section{NATIONAL INSTITUTE OF ADVANCED SCIENCE AND TECHNOLOGY}

\section{COLLABORATION SCORE WITH INDUSTRY: 50}

It's notoriously difficult to make a material that reflects light and is also thermally insulating.

s. In August last year a collaboration between researchers from the National Institute of Advanced Industrial Science and Technology (AIST) and Kawaken Fine Chemicals Co. Ltd succeeded where others had failed.

Taking inspiration from the layered arrangement of fish scales, the research team devised a simple method for producing an alumina film that strongly reflects light and has a high thermal insulation comparable to that of wool. The method was reported in Advanced Materials.

The study demonstrates the importance of fostering collaborations across the academiaindustry divide says Kenji Hata, the director of the CNT-Application Research Center at AIST.

"Ten or twenty years ago, Japanese companies were very strong and would hire their own researchers, but they can't afford to do that anymore."

AIST was established in 2001 with a strong emphasis on applied science and collaborations with industry. The institution underwent a major reorganization in 2015 to better align its projects to meet the needs of industry, including creating a new organization in charge of technology marketing. Today, in addition to its own 2,300 researchers, about 1,800 scientists and engineers from private industry carry out research at AIST each year.

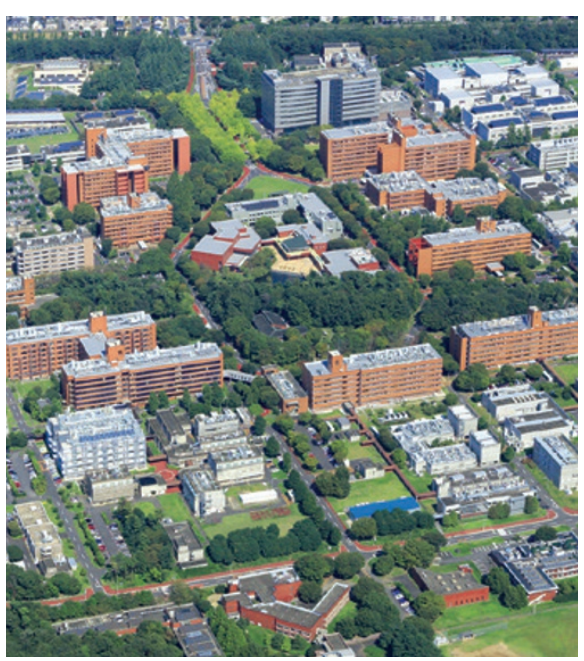

AIST campus in Tsukuba

In 2015, AIST's most fruitful industry partnership in the Nature Index was microscope manufacturer JEOL Ltd, followed by telephone communications company, NTT Group. -

\section{AIST'S PARTNERSHIPS}

AIST'S top 5 international collaboration hotspots; and top 5 collaborating institutions.
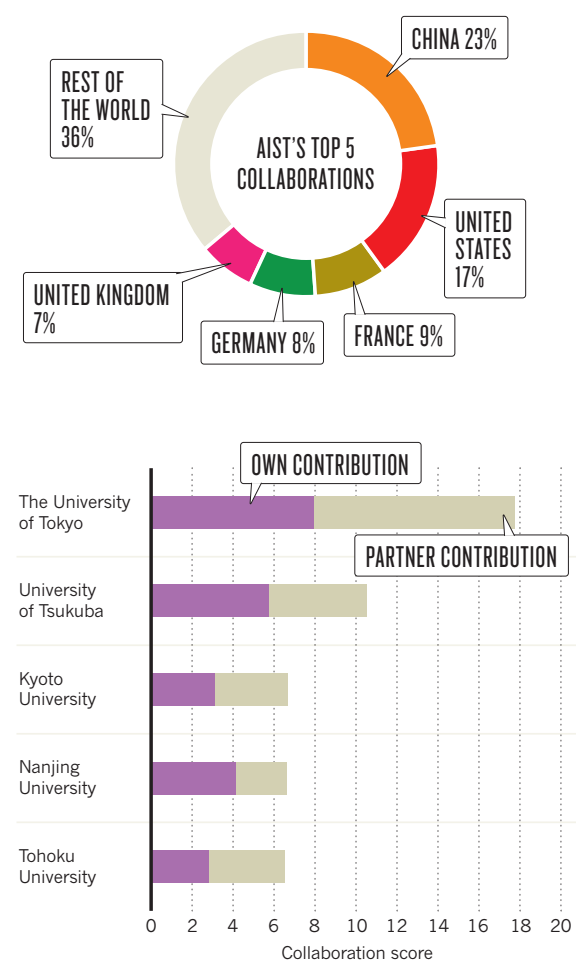